\title{
Locally advanced pancreatic cancer: staging, operability, and the importance of multimodality therapy
}

\author{
Rebecca Y. Kim, Douglas B. Evans, Kathleen K. Christians \\ Department of Surgery and the LaBahn Pancreatic Cancer Program, Medical College of Wisconsin, Milwaukee, WI, USA \\ Correspondence to: Kathleen K. Christians, MD. Department of Surgery, Medical College of Wisconsin, 8701 West Watertown Plank Road, \\ Wauwatosa, WI 53226, USA. Email: kchristi@mcw.edu. \\ Provenance and Peer Review: This article was commissioned by the editorial office, Hepatobiliary Surgery and Nutrition. The article did not undergo \\ external peer review. \\ Comment on: Gemenetzis G, Groot VP, Blair AB, et al. Survival in Locally Advanced Pancreatic Cancer After Neoadjuvant Therapy and Surgical \\ Resection. Ann Surg 2019;270:340-7.
}

Submitted Oct 31, 2019. Accepted for publication Nov 12, 2019.

doi: 10.21037/hbsn.2019.11.26

View this article at: http://dx.doi.org/10.21037/hbsn.2019.11.26

The role for surgery in the management of locally advanced pancreatic cancer (LAPC) is nicely discussed in the manuscript by Gemenetzis et al. published in the Annals of Surgery (1). As the authors acknowledge, therapeutic selection, sequencing and surgical resection were based on expert consensus in their multidisciplinary clinic. The excellent results achieved by these authors reflect their experience and expertise; both qualities which may be hard to replicate in smaller volume programs. The challenge in treating patients with LAPC is demonstrated by the inability of most clinicians and radiologists to develop an objective working definition of LAPC. Even professional societies and consensus panels struggle to agree on a computed tomography- (CT) or magnetic resonance image (MRI)-based definition of LAPC (2-4). In the absence of an objective, reproducible clinical staging system for localized pancreatic cancer (PC), it becomes very hard for the individual medical or surgical oncologist to translate published treatment results to the newly diagnosed patient.

In an effort to add objectivity to the staging of localized PC, we have published a CT-based system utilizing critically important tumor vessel relationships (5-7). We defined two anatomically distinct disease patterns summarized in Table 1. In general, patients with LAPC type A have greater than 180 degrees of tumor-vessel contact but less than 270 degrees, which allows for dissection (with tumorartery separation) or resection of the celiac and/or common hepatic artery (CHA), or dissection of the perineurium from the adventitia of the superior mesenteric artery (SMA). If the patient with localized, non-metastatic PC is deemed acceptable for surgery (based on performance status, medical co-morbidities, social support), the clinical disease stage determined by cross-sectional imaging at diagnosis will accurately predict the likelihood of completing all intended neoadjuvant therapy and surgery (7-10). To the extent that surgery is necessary (even if not sufficient) for long term survival, this information is invaluable for patients, their family, and their treating physicians. Consistent with the results reported by Gemenetzis and colleagues, we demonstrated over two decades ago that neoadjuvant treatment sequencing accurately dichotomizes patients with localized PC into those who will and will not achieve a clinical benefit from surgical resection of their primary tumor (11). If treatment success includes the completion of all intended neoadjuvant therapy and surgery, contemporary data from our institution suggests that such success is stage dependent; for patients with resectable, borderline resectable, locally advanced type A and locally advanced type B PC, the likelihood of completing all intended neoadjuvant therapy and surgery is $90 \%, 75 \%$, $60 \%$ and $25 \%$ respectively (6-10). Clearly, as systemic therapies continue to improve, so will survival duration.

Another subject within the field of LAPC is the issue of tumor resectability - how is this determined pre-operatively and intra-operatively, and who should be considered for surgery? Gemenetzis and colleagues comment that imaging 
Table 1 Medical College of Wisconsin LAPC Classification based on objective tumor-vessel relationships as seen on high quality cross-sectional imaging

\begin{tabular}{|c|c|c|}
\hline Vessel & \multicolumn{2}{|c|}{ Tumor relationship } \\
\hline \multirow[t]{2}{*}{ CA } & $>180^{\circ}$ & $>180^{\circ}$ \\
\hline & $\begin{array}{l}\text { No extension to the aorta } \\
\text { Amenable to celiac resection }\end{array}$ & Abutment/encasement of the aorta \\
\hline SMA & $>180^{\circ}$ but $\leq 270^{\circ}$ & $>270^{\circ}$ \\
\hline $\mathrm{CHA}$ & $\begin{array}{l}\text { Extension to CA that is amenable to } \\
\text { reconstruction }\end{array}$ & $\begin{array}{l}\text { Extension to bifurcation of left and right hepatic } \\
\text { arteries }\end{array}$ \\
\hline SMV/PV & $\begin{array}{c}\text { Occlusion, but distal target }>1.5 x \text { SMA } \\
\text { diameter }\end{array}$ & $\begin{array}{l}\text { Occlusion without obvious option for venous } \\
\text { reconstruction }\end{array}$ \\
\hline
\end{tabular}

LAPC, locally advanced pancreatic cancer; CA, celiac axis; SMA, superior mesenteric artery; CHA, common hepatic artery; SMV, superior mesenteric vein; PV, portal vein; GDA, gastroduodenal artery; LGA, left gastric artery.

response following neoadjuvant therapy may be inadequate to declare resectability, implying that a more aggressive view towards proceeding to the operating room is justified in this current era of more effective systemic therapy (mFOLFIRINOX). We caution most surgeons against adopting this philosophy as $20(17 \%)$ patients received an operation which conveyed no therapeutic benefit (judged unresectable at the time of laparotomy). Given the experience and international reputation of the authors, one would assume that $17 \%$ represents the absolute lowest end of the range achievable by less experienced surgeons. An alternative way to think about this issue is to reflect on the anatomy of the visceral arteries which are surrounded by a perineural sheath that is interposed between the tumor and the adventitia of the artery. In some cases, the surgeon can develop the plane of dissection between the adventitia and the surrounding perineurium, preserving the artery despite preoperative imaging that suggested tumor encasement. Conversely, if this plane of dissection cannot be developed due to tumor infiltration of the autonomic perineural sheath, the artery will need to be replaced. Local tumor unresectability may become more common in this era of more effective non-surgical therapies-patients previously deemed unresectable based on preoperative imaging may then be "explored" in the hope of separating the visceral artery from the tumor. We would advise surgeons to reserve operations for patients in whom they are equipped to resect and reconstruct the artery in question, rather than hope that the perineum can be separated from the adventitia. We agree that currently, preoperative imaging cannot definitively confirm for the surgeon whether or not this plane will be successfully developed. Importantly, our objective CT-criteria for recommending surgery (resectability/operability) after neoadjuvant therapy assumes the ability to resect the celiac or hepatic arteries if they cannot be separated from the surrounding perineurium and the ability to restore venous return from the midgut (suitable proximal and distal target for venous reconstruction) $(7,12,13)$.

The optimal delivery of neoadjuvant therapy includes accurate pretreatment staging, an objective system for preoperative determination of operability (which tumors are removeable and which are not) and importantly, a mechanism to assess the oncologic outcome of the treatment delivered. Assessment of treatment response is critically important to avoid operating on patients who are destined to early recurrence. While we are exploring a variety of blood-based biomarkers and imaging correlates, carbohydrate antigen 19-9 (Ca19-9) remains a readily available biomarker which is elevated at diagnosis in the majority of PC patients. We have recently demonstrated that patients with pretreatment Ca19-9 levels of $<80 \mathrm{U} / \mathrm{mL}$ 
are more likely to successfully complete all intended neoadjuvant therapy, undergo successful surgical resection, have a lower pathologic stage, and have the most favorable overall survival (14). In fact, normalization of Ca19-9 following neoadjuvant therapy is associated with a doubling of overall survival in both the preoperative (24-46 months) and postoperative (20-43 months) setting compared to patients who fail to achieve such a decline in Ca19-9. We, therefore, monitor Ca19-9 at diagnosis once the bilirubin has normalized and at each restaging interval. In LAPC patients who fail to experience a decline in Ca19-9 after the first two months of induction system therapy, we consider a change in chemotherapeutic agents. The best-case scenario is for patients to have their Ca19-9 normalize at the end of neoadjuvant therapy just prior to surgery. To increase the likelihood of achieving this result we explored the value of molecular profiling biopsy specimens to personalize neoadjuvant therapy (10). In so doing, greater than $80 \%$ of patients were able to complete all intended neoadjuvant therapy and surgery with a median overall survival of 45 months for patients with resected PC. As molecular phenotyping of tumors and other scientific advances continue to evolve, we are likely to see this technology utilized in patients with LAPC.

Also demonstrated in the manuscript by Gemenetzis and colleagues, operations for LAPC are some of the most technically complex and difficult in pancreas surgery and are associated with significant risk for major morbidity and mortality. For these reasons, our group developed a readily translatable, easily reproducible staging system for LAPC based on high quality pretreatment imaging (7). Although induction therapy may diminish the tumor volume/ diameter, it rarely changes the tumor-artery interface. In LAPC type B disease, complete resection without significant response to neoadjuvant therapy would require either cutting through tumor or a vascular reconstruction that is not technically feasible (lacks proximal or distal venous target). Examining 96 consecutive patients with LAPC who were treated with an extended course of neoadjuvant chemotherapy and intensity-modulated radiation therapy (IMRT), we demonstrated that those patients with type A disease were more likely to undergo surgical resection following neoadjuvant therapy compared to patients with type B disease (62\% versus $24 \%$, respectively). Overall survival reflected successful completion of neoadjuvant therapy and surgical resection (55.6 versus 37.5 months for type A and type B disease, respectively). The patients with type B LAPC who underwent successful surgical resection of the primary tumor achieved a fairly dramatic response to induction therapy. Some of this radiographic response likely included the resolution of inflammatory change which can complicate the interpretation of initial CT images at the time of diagnosis.

In summary, the landscape for LAPC is clearly changing as nicely demonstrated in the manuscript by Gemenetzis and colleagues. Success in the delivery of neoadjuvant therapy that then culminates in surgical resection is stage dependent. Resectability is best determined preoperatively and an operation should only be undertaken if the surgeon is equipped to resect and reconstruct the involved vessel(s). The use of CT imaging criteria at diagnosis allows for LAPC patients to be placed in two distinct stages of disease (A and $\mathrm{B}$ ) allowing for prognostication of both the success of planned treatment as well as overall survival. Surgery in patients with LAPC should be considered a potentially valuable option in very carefully selected patients. Knowing when and how to integrate surgery is an evolving clinical and translational question best approached with the humility that characterizes our impact on this challenging disease.

\section{Acknowledgments}

Funding: None.

\section{Footnote}

Conflicts of Interest: All authors have completed the ICMJE uniform disclosure form (available at http://dx.doi. org/10.21037/hbsn.2019.11.26). The authors have no conflicts of interest to declare.

Etbical Statement: The authors are accountable for all aspects of the work in ensuring that questions related to the accuracy or integrity of any part of the work are appropriately investigated and resolved.

Open Access Statement: This is an Open Access article distributed in accordance with the Creative Commons Attribution-NonCommercial-NoDerivs 4.0 International License (CC BY-NC-ND 4.0), which permits the noncommercial replication and distribution of the article with the strict proviso that no changes or edits are made and the original work is properly cited (including links to both the formal publication through the relevant DOI and the license). See: https://creativecommons.org/licenses/by-nc-nd/4.0/. 


\section{References}

1. Gemenetzis G, Groot VP, Blair AB, et al. Survival in Locally Advanced Pancreatic Cancer After Neoadjuvant Therapy and Surgical Resection. Ann Surg 2019;270:340-7.

2. Seufferlein T, Hammel P, Delpero JR, et al. Optimizing the management of locally advanced pancreatic cancer with a focus on induction chemotherapy: Expert opinion based on a review of current evidence. Cancer Treat Rev 2019;77:1-10.

3. NCCN. Pancreatic Adenocarcinoma. Version 3.2019. NCCN Clinical Practice Guidelines in Oncology. National Comprehensive Cancer Network; 2019. Available online: https://www.nccn.org/professionals/physician_gls/ pdf/pancreatic.pdf

4. Isaji S, Mizuno S, Windsor JA, et al. International consensus on definition and criteria of borderline resectable pancreatic ductal adenocarcinoma 2017. Pancreatology 2018;18:2-11.

5. Evans DB. What Makes a Pancreatic Cancer Resectable? Am Soc Clin Oncol Educ Book 2018;38:300-5.

6. Evans DB, Kamgar M, Tsai S. Goals of Treatment Sequencing for Localized Pancreatic Cancer. Ann Surg Oncol 2019;26:3815-9.

7. Chatzizacharias NA, Tsai S, Griffin M, et al. Locally advanced pancreas cancer: Staging and goals of therapy.

Cite this article as: Kim RY, Evans DB, Christians KK. Locally advanced pancreatic cancer: staging, operability, and the importance of multimodality therapy. HepatoBiliary Surg Nutr 2020;9(4):497-500. doi: 10.21037/hbsn.2019.11.26
Surgery 2018;163:1053-62.

8. Christians KK, Heimler JW, George B, et al. Survival of patients with resectable pancreatic cancer who received neoadjuvant therapy. Surgery 2016;159:893-900.

9. Barnes CA, Chavez MI, Tsai S, et al. Survival of patients with borderline resectable pancreatic cancer who received neoadjuvant therapy and surgery. Surgery 2019;166:277-85.

10. Tsai S, Christians KK, George B, et al. A Phase II Clinical Trial of Molecular Profiled Neoadjuvant Therapy for Localized Pancreatic Ductal Adenocarcinoma. Ann Surg 2018;268:610-9.

11. Evans DB, Rich TA, Byrd DR, et al. Preoperative chemoradiation and pancreaticoduodenectomy for adenocarcinoma of the pancreas. Arch Surg 1992;127:1335-9.

12. Chavez MI, Tsai S, Clarke CN, et al. Distal splenorenal and mesocaval shunting at the time of pancreatectomy. Surgery 2019;165:298-306.

13. Christians KK, Pilgrim CH, Tsai S, et al. Arterial resection at the time of pancreatectomy for cancer. Surgery 2014;155:919-26.

14. Tsai S, George B, Wittmann D, et al. Importance of Normalization of CA19-9 Levels Following Neoadjuvant Therapy in Patients With Localized Pancreatic Cancer. Ann Surg 2020;271:740-7. 